\title{
Lower Respiratory System
}

National Cancer Institute

\section{Source}

National Cancer Institute. Lower Respiratory System. NCI Thesaurus. Code C33012.

The part of the respiratory system below the bifurcation of the trachea. It includes the lungs and the parts of the lungs such as the bronchioles and alveoli. 